\title{
Epidemiology of Maxillofacial Fracture - A Hospital Based Study
}

\author{
Mahat $A K^{1}$, Gurung $G 2$, Shrestha $M^{3}$, Chaudhary $B^{4}$
}

\section{ABSTRACT}

Introduction: Maxillofacial trauma is the most common problem seen by maxillofacial surgeons in Nepal. The causes of facial fractures are road traffic accidents (RTA), assaults, falls, gunshot wounds, domestic violence, sports and work injuries. Maxillofacial injury varies from soft tissue injury to facial bone fractures. This study was conducted to assess the burden of maxillofacial trauma in mid-western and far-western regions of Nepal. Material and Method: Patients with maxillofacial fractures admitted in dental ward of NGMCTH, Kohalpur, from April 2017 to April 2019 were included in the study. Patients having soft tissue injury without facial bone fractures and OPD cases were excluded from the study. Patient demographics, mechanism of injury, associated soft tissue injury, accompanying injuries to other parts of body and facial bone fractures were recorded. Patient were treated by open reduction internal fixation or lateral compression splint with circum-mandibular wiring or managed conservatively as necessary. Result: A total of 87 patients were included in the study. The age of patient ranged from 2 years to 67 years with a mean of 26.87 years. Most of the injuries occurred between the age group of 20-29 years with male: female ratio 5.2:1. The most frequent cause of facial bone fracture was RTA (51.7\%) and the most frequently fractured facial bone was mandible. Contusion $54(49.5 \%)$ was the most common associated soft tissue injury. Head injury 20(52.6\%) was the most common accompanying injury to other parts of body. The mean duration of hospital stay was 6.29. Conclusion: Male patient in their third decade mostly had mandible fracture due to road traffic accident. Head injury was the most common accompanying injury. Contusion was the most common type of associated soft tissue injury.

\section{Key words: Associated injuries, Epidemiology, Maxillofacial fracture}

1. Dr. Arun Kumar Mahat

2. Dr. Gopal Gurung

3. Dr. Merina Shrestha

4. Dr. Bishwanath Chaudhary

\section{Address for Correspondence:}

\author{
Dr. Arun Kumar Mahat \\ Lecturer \\ Department of Dentistry \\ Nepalgunj Medical College \& Teaching Hospital \\ Kohalpur, Banke \\ E-mail:dr.arunmahat@gmail.com
}

\section{INTRODUCTION}

Maxillofacial trauma is the most common problem seen by maxillofacial surgeons in Nepal. The epidemiology of maxillofacial injury varies on several factors such as patients' demography, geographical area, culture, lifestyle differences, socioeconomic trends, road safety regulations and alcohol abuse $e^{1,2,3,4,5}$. The causes of facial fractures are road traffic accidents (RTA), assaults, falls, gunshot wounds, domestic violence, sports and work injuries ${ }^{2,3,4}$. Maxillofacial injury varies from soft tissue injury (e.g. abrasion, contusion, laceration) to facial bone fractures. Patients presenting with maxillofacial fractures may also have accompanying injuries such as head injury, spinal injury, intra-thoracic injury, intraabdominal injuries, limb fractures etc ${ }^{6,7}$. The treatment option varies from conservative management, closed method to surgical measures and is associated with various complications such as: infected metal ware, malocclusion, malunion, plate exposure, palpable plate, wound infection, abnormal facial contour requiring recontouring and nonunion. There may be enophthalmos, blindness, facial dysaesthesia, temporomandibular joint (TMJ) dysfunction, dento-alveolar, lachrymal, sinonasal or airway pathologies and central nervous system (CNS) complications (e.g., cerebrospinal fluid (CSF) leak, meningitis, cerebral bleed or abscess) $)^{5}$. Similar studies in our countries had shown male predominance and RTA to be the most common cause of injuries ${ }^{8,9,10,11}$. Mandibular parasymphysis was the most common site of mandibular fracture ${ }^{10,11}$. Study by Yadav S. showed extradural hematoma (EDH) to be the most common type of head injury associated with the maxillofacial injury ${ }^{9}$. There are no study evaluating the trauma burden till date done in mid-western and farwestern region of Nepal. Thus it is necessary to understand the trauma burden and to help develop more efficient ways to plan resource allocation and to deliver care and preventive measures. So, we designed a descriptive observational study to obtain a reliable data on demographics, causes, associated injuries and length of hospital stay in patient with maxillofacial injury so that an injury prevention strategy can be developed and patients at risk of developing postoperative complications can be identified. 


\section{MATERIAL AND METHOD}

Nepalgunj Medical College Teaching Hospital (NGMCTH) is the tertiary care hospital and the only referral center for maxillofacial injuries in mid-western and far-western region of Nepal. Patients with maxillofacial fractures admitted in dental ward of NGMCTH, Kohalpur, from April 2017 to April 2019 were included in the study. Patients having soft tissue injury without facial bone fractures and OPD cases were excluded from the study. Patient demographics, mechanism of injury, associated soft tissue injuries, accompanying injuries to other parts of body and facial bone fractures were recorded. Patient were treated by open reduction internal fixation or lateral compression splint with circum-mandibular wiring or managed conservatively as necessary. The data were entered in Microsoft Excel 2007 and analyzed using Statistical Package for Social Sciences (SPSS) version 11.5.

\section{RESULT}

A total of 87 patients were treated for maxillofacial fracture in Nepalgunj Medical College Teaching Hospital, Kohalpur, Banke, Nepal during the study period. The age group of patient ranged from 2 years to 67 years with a mean age of 26.87 years. Most of the injuries $30(34.5 \%)$ occurred between the age group of $20-29$ years. There were 73 males and 14 females with a male: female ratio 5.2:1. The most frequent cause of facial bone fracture was RTA $51.7 \%$ (45/87) followed by fall injury $20.7 \%$ (18/87), physical assault $14.9 \%(13 / 87)$, occupational injuries $4.6 \%(4 / 87)$, stumbling $3.5 \%(3 / 87)$, animal attack $1.1 \%(1 / 87)$, sports injuries $2.3 \%(2 / 87)$ and domestic violence $1(1.1 \%)$. Among RTA motorbike accident (28/45) was the most common cause. (Table I)

\begin{tabular}{|c|c|c|c|c|c|c|c|c|c|c|c|c|c|c|}
\hline \multirow[b]{2}{*}{ 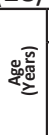 } & \multicolumn{4}{|c|}{ RTA } & \multicolumn{3}{|c|}{ Fall } & \multirow[b]{2}{*}{ 琶) } & \multirow[b]{2}{*}{ 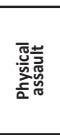 } & \multirow[b]{2}{*}{ 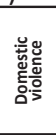 } & \multirow[b]{2}{*}{ 厗 } & \multirow[b]{2}{*}{ 䇏 } & \multirow[b]{2}{*}{ 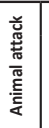 } & \multirow[b]{2}{*}{ 愛 } \\
\hline & $\underline{\Sigma}$ & 㩊 & 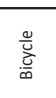 & 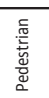 & 总 & 高 & 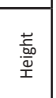 & & & & & & & \\
\hline के & 。 & $\circ$ & 。 & ح高 & 。 & N鯂 & 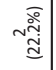 & 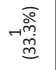 & 。 & 。 & 。 & 。 & $\circ$ & 卜 \\
\hline 尊 & 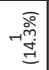 & 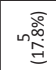 & 零 & ح高 & 皮 & - & 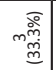 & 。 & 承 & o & 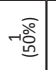 & ᄀ. & 0 & 㥠 \\
\hline ণ্రి & 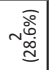 & 墙 & 霘 & 。 & ন ন & 。 & 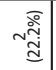 & 。 & 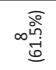 & ○ & r吾 & 。 & $\circ$ & 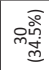 \\
\hline 商 & 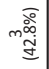 & 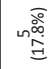 & $\circ$ & ন ন & ন & 0 & 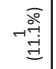 & 。 & m. & 。 & $\circ$ & 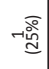 & 0 & H: \\
\hline $\begin{array}{l}\text { go } \\
\text { o }\end{array}$ & 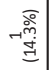 & 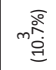 & 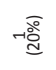 & 0 & 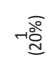 & 嵪 & - & 。 & 。 & - & $\circ$ & ন & 0 & 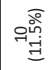 \\
\hline 絗 & 0 & & 。 & 。 & 。 & 。 & 0 & 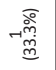 & 。 & 。 & 0 & ন & 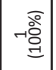 & 屆 \\
\hline$\stackrel{8}{i}$ & 。 & $\circ$ & 0 & 。 & $\circ$ & $\circ$ & 。 & 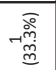 & ○ & 。 & $\circ$ & o & 0 & 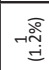 \\
\hline 営 & 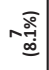 & 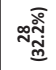 & 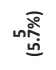 & 的高 & 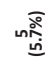 & نَّ & 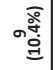 & $\begin{array}{l}\text { 㝵 } \\
\text { min }\end{array}$ & 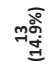 & ન & 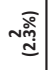 & 我 & 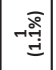 & ळo్తి \\
\hline \multicolumn{15}{|c|}{ Gender } \\
\hline$\frac{\frac{\alpha}{\omega}}{\Sigma}$ & 丞 & 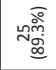 & 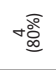 & m.্ట్ & m: & m måํㄹㄹ & 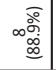 & $\begin{array}{l}\text { 高 } \\
\text { 胥 } \\
\underline{\underline{G}}\end{array}$ & mర్తి & 。 & r总 & + & 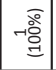 & 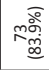 \\
\hline 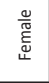 & 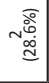 & 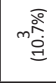 & r胥 & 八高 & 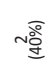 & 一选 & | & 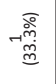 & 。 & 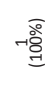 & 。 & 。 & 。 & 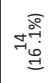 \\
\hline 咢 & 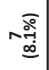 & 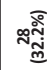 & 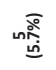 & 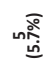 & 周 & 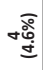 & 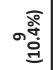 & 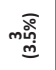 & 狷 & ન. & 疍 & 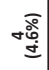 & 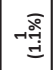 & ळo్త్తి \\
\hline
\end{tabular}

Table I: Age and Gender distribution of patients according to mechanism of injury
Legends: RTA (Road traffic accident), MVA (Motor vehicle accident), MBA (Motorbike accident)

Mandible fracture occurred in 68 cases. Among them, 32 cases had mandible fracture in single site, 20 cases had mandible fracture at multiple sites and 16 cases had mandible fracture in combination with other facial bone fracture. Mandible was fractured at 97 anatomic locations. Mandibular parasymphysis (39/97) was the most common site of fracture with right: left ratio of 11:28 followed by angle of mandible 22/97 (R:L= 13:9), symphysis $13 / 97$, body $11 / 97$ (R:L=7:4), condyle 10/97 ( $R: L=6: 4)$, ramus $2 / 97$. RTA mostly resulted in mandibular parasymphysis fracture whereas; physical assault mostly resulted in mandibular angle fracture (Table II).

\begin{tabular}{|c|c|c|c|c|c|c|c|c|c|c|c|c|c|c|}
\hline \multirow{2}{*}{ 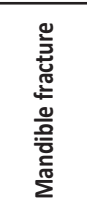 } & \multicolumn{4}{|c|}{ RTA } & \multicolumn{3}{|c|}{ Fall } & \multirow[b]{2}{*}{$\mid$} & \multirow{2}{*}{ 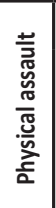 } & \multirow{2}{*}{ 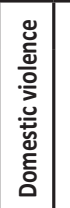 } & \multirow[b]{2}{*}{$\begin{array}{l}\text { 는 } \\
\text { 음 }\end{array}$} & \multirow[b]{2}{*}{ 产 } & \multirow[b]{2}{*}{ 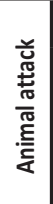 } & \multirow[b]{2}{*}{ 푬 } \\
\hline & $\sum_{\Sigma}^{\mathbb{S}}$ & 高 & 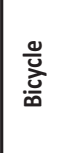 & 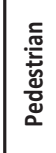 & 志 & ¿ఃे & 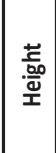 & & & & & & & \\
\hline 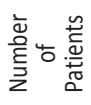 & 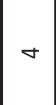 & $\stackrel{\text { N }}{\text { s. }}$ & in & $\sigma$ & n & $m$ & $a$ & $m$ & $\approx$ & $\rightarrow$ & $\rightarrow$ & $\rightarrow$ & 0 & 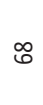 \\
\hline 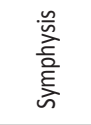 & $\rightarrow$ & n & 0 & 0 & $\neg$ & $\rightarrow$ & $\sim$ & 0 & $\sim$ & 0 & 0 & $\rightarrow$ & 0 & $\stackrel{m}{\rightarrow}$ \\
\hline 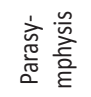 & 9 & $\underset{\stackrel{\mathscr{\sigma}}{G}}{\stackrel{\omega}{G}}$ & $\overrightarrow{\widetilde{s}}$ & $\simeq$ & $\overrightarrow{\vec{v}}$ & $\simeq$ & 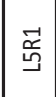 & 9 & $\underset{\widetilde{I}}{\widetilde{J}}$ & $\vec{x}$ & $\exists$ & 0 & 0 & gి \\
\hline 흥 & $\vec{x}$ & $\approx$ & $\vec{g}$ & 0 & $\simeq$ & 0 & $\overrightarrow{\vec{s}}$ & 0 & $\simeq$ & ○ & $\circ$ & 0 & o & $\Rightarrow$ \\
\hline$\frac{\frac{0}{00}}{\frac{00}{\alpha}}$ & $\exists$ & $\stackrel{\dddot{m}}{\stackrel{m}{m}}$ & $\approx \widetilde{\simeq}$ & $\underset{\sim}{\vec{\exists}}$ & $\vec{x}$ & 0 & $\vec{\sim}$ & 0 & $\underset{\stackrel{J}{J ~}}{\stackrel{J}{J}}$ & $\exists$ & $\vec{x}$ & 0 & 0 & $\approx$ \\
\hline 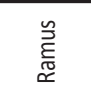 & 0 & 0 & 0 & 0 & 0 & 0 & $\vec{x}$ & 0 & $\vec{x}$ & 0 & 0 & 0 & 0 & $\sim$ \\
\hline $\begin{array}{l}\frac{0}{\vec{z}} \\
\overline{0}\end{array}$ & 0 & $\exists$ & 0 & $\underset{\sim}{\vec{\mu}}$ & $\overrightarrow{\vec{a}}$ & $\vec{x}$ & 0 & $\approx$ & $\exists$ & 0 & 0 & 0 & 0 & 음 \\
\hline $\begin{array}{l}\text { 음 } \\
\text { 밈 } \\
\end{array}$ & 0 & 0 & 0 & 0 & 0 & 0 & 0 & 0 & 0 & 0 & 0 & 0 & 0 & 0 \\
\hline 覀 & 0 & $\stackrel{\Perp}{ }$ & 0 & 0 & 웅 & $\sigma$ & $m$ & 0 & $\stackrel{\varphi}{\circ}$ & $\sim$ & $\sim$ & $\vec{r}$ & 0 & ธิ \\
\hline
\end{tabular}

Table II: Pattern of mandibular fracture according to the etiology of injury

Legends: RTA= Road traffic accident, Motor vehicle accident (MVA), MBA (Motorbike accident), ZMC=Zygomatico maxillary complex, $\mathrm{NOE}=$ Naso-orbitoethmoid. $\mathrm{R}=$ Right, $\mathrm{L}=$ Left

Maxilla is the most frequently fracture bone in mid-facial region. Zygomatico-maxillary complex (ZMC) fracture occurred in 15 cases, unilateral maxilla in 14 cases, palatal split occurring in 12 cases, nasal bone in 6 cases. We reported one case with frontal sinus fracture and naso-orbito-ethmoid fracture. We reported slightly higher frequency of Lefort III fracture (4 cases) compared to Lefort I and Lefort II fracture (Table III). 


\begin{tabular}{|c|c|c|c|c|c|c|c|c|c|c|c|c|c|c|}
\hline \multirow{2}{*}{ 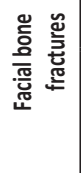 } & \multicolumn{4}{|c|}{ RTA } & \multicolumn{3}{|c|}{ Fall } & \multirow{2}{*}{ 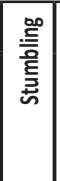 } & \multirow{2}{*}{ 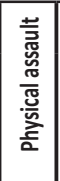 } & \multirow{2}{*}{ 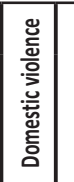 } & \multirow{2}{*}{ 善 } & \multirow{2}{*}{ 产 } & \multirow{2}{*}{ 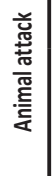 } & \multirow[t]{2}{*}{\begin{tabular}{|l} 
㞼 \\
\end{tabular}} \\
\hline & $\underline{\Sigma}$ & $\stackrel{\Phi}{\mathbf{\Phi}}$ & 递 & 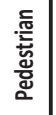 & $\stackrel{\Xi}{\stackrel{\Xi}{2}}$ & "ᄒ⿱宀 & 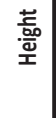 & & & & & & & \\
\hline 㲋 - & 0 & $m$ & 0 & 0 & 0 & 0 & 0 & 0 & 0 & 0 & 0 & 0 & 0 & $m$ \\
\hline $\begin{array}{l}\frac{\mathrm{t}}{\mathrm{t}} \\
\frac{\mathrm{Q}}{\mathrm{S}}\end{array}=$ & 0 & 0 & 0 & - & 0 & $\rightarrow$ & $\rightarrow$ & 0 & 0 & 0 & 0 & 0 & 0 & $m$ \\
\hline 䓂 $\equiv$ & 0 & $m$ & 0 & 0 & 0 & $\rightarrow$ & 0 & 0 & 0 & 0 & 0 & ○ & 0 & + \\
\hline$\sum_{N}^{u}$ & 芩 & $\stackrel{m}{\widetilde{x}}$ & 0 & $\vec{x}$ & 0 & $\exists$ & $\overrightarrow{\underline{\Xi}}$ & 0 & 0 & 0 & $\vec{x}$ & $\overrightarrow{\vec{\alpha}}$ & 0 & 옴 \\
\hline 岁 & $\rightarrow$ & $\rightarrow$ & 0 & 0 & 0 & 0 & 0 & 0 & 0 & 0 & 0 & 0 & 0 & $\sim$ \\
\hline $\begin{array}{l}\overline{\widetilde{g}} \\
\overline{\check{m}}\end{array}$ & $\rightarrow$ & $\rightarrow$ & 0 & 0 & $\rightarrow$ & $\sim$ & 0 & 0 & 0 & 0 & 0 & $\rightarrow$ & 0 & 0 \\
\hline $\begin{array}{l}\frac{7}{\frac{7}{5}} \\
\frac{\pi}{2}\end{array}$ & 0 & $\sigma$ & $\rightarrow$ & 0 & 0 & $\rightarrow$ & $\rightarrow$ & 0 & - & 0 & 0 & $m$ & $\rightarrow$ & $\approx$ \\
\hline 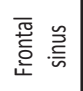 & 0 & 0 & 0 & 0 & 0 & 0 & 0 & 0 & 0 & 0 & $\circ$ & $\vec{x}$ & 0 & $\rightarrow$ \\
\hline 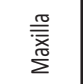 & $\simeq$ & $\underset{\vec{\alpha}}{\vec{\partial}}$ & $\exists$ & 0 & 0 & 0 & 0 & 0 & $\overrightarrow{\vec{\alpha}}$ & 0 & ○ & $\underset{\vec{\alpha}}{\approx}$ & $\exists$ & 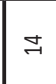 \\
\hline
\end{tabular}

Table III: Pattern of mid-face fracture according to the etiology of injuries

Out of 87 cases, 33 patients sustained accompanying injuries. Head injuries occurred in 20 patients. Extremities were injured in 9 patients. C-spine injury was noted in 5 patients with fall from tree being the most common cause ( 2 patients). Chest trauma and abdominal injuries occurred in 2 patients each (Table IV)

\begin{tabular}{|c|c|c|c|c|c|c|c|c|c|c|c|c|c|c|}
\hline \multirow{2}{*}{ 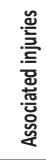 } & \multicolumn{4}{|c|}{ RTA } & \multicolumn{3}{|c|}{ Fall } & \multirow[b]{2}{*}{ 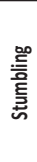 } & \multirow{2}{*}{ 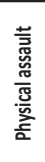 } & \multirow{2}{*}{ 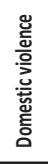 } & \multirow[b]{2}{*}{ 言 } & \multirow[b]{2}{*}{ 产 } & \multirow{2}{*}{ 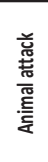 } & \multirow[b]{2}{*}{ 舘 } \\
\hline & $\underline{\Sigma}$ & 诚 & 营 & 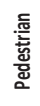 & 总 & 'ᄒ̀ & $\begin{array}{l}\text { 器 } \\
\text { 产 }\end{array}$ & & & & & & & \\
\hline 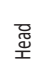 & 总 & $\begin{array}{l}\text { 옹 } \\
\stackrel{\text { no }}{n}\end{array}$ & 0 & 庐 & 总 & 힣 & 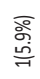 & 0 & 0 & 总 & 0 & $\begin{array}{l}\text { 总 } \\
\stackrel{\vec{g}}{m}\end{array}$ & 0 & 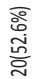 \\
\hline 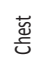 & 0 & 0 & 0 & 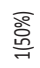 & 0 & 0 & 0 & 0 & 0 & 0 & 0 & $\begin{array}{l}\text { 总 } \\
\text { 兽 }\end{array}$ & 0 & $\begin{array}{l}\text { 总 } \\
\stackrel{\text { D. }}{\sim}\end{array}$ \\
\hline 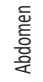 & $\begin{array}{l}\text { बें } \\
\text { 음 }\end{array}$ & 0 & 0 & 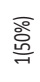 & 0 & 0 & 0 & 0 & 0 & 0 & 0 & 0 & 0 & 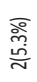 \\
\hline 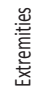 & & 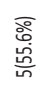 & 0 & 0 & 0 & 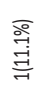 & 0 & 0 & 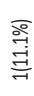 & 0 & 0 & 0 & $\begin{array}{l}\text { 월 } \\
\text { ت्ञ }\end{array}$ & 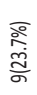 \\
\hline $\begin{array}{l}\text { 产 } \\
\text { d. }\end{array}$ & 0 & 0 & 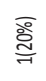 & 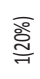 & 高 & 0 & 0 & 0 & 0 & 0 & 0 & $\begin{array}{l}\text { 웡 } \\
\text { (n) }\end{array}$ & 0 & 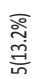 \\
\hline 呇 & 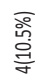 & 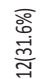 & 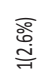 & $\begin{array}{l}\text { बे } \\
\text { ò } \\
\text { 빙 }\end{array}$ & $\begin{array}{l}\overline{\text { बे }} \\
\stackrel{\bar{m}}{m}\end{array}$ & $\begin{array}{l}\text { 오 } \\
\stackrel{一}{m}\end{array}$ & 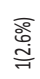 & 0 & $\begin{array}{l}\text { 용 } \\
\stackrel{ }{-1}\end{array}$ & $\begin{array}{l}\text { बें } \\
\stackrel{ }{\sim}\end{array}$ & 0 & 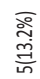 & $\begin{array}{l}\overline{\mathrm{o}} \\
\stackrel{\mathrm{d}}{\mathrm{j}}\end{array}$ & 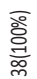 \\
\hline
\end{tabular}

Table IV: Pattern of accompanying injuries to other parts of body according to the mechanism of injuries

Contusion was the most common associated soft tissue injury with facial bone fracture (Table V).

\begin{tabular}{|c|c|c|c|c|c|c|c|c|c|c|c|c|c|c|}
\hline \multirow{2}{*}{ 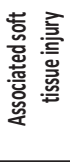 } & \multicolumn{4}{|c|}{ RTA } & \multicolumn{3}{|c|}{ Fall } & \multirow{2}{*}{ 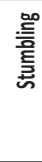 } & \multirow{2}{*}{ 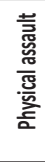 } & \multirow{2}{*}{ 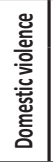 } & \multirow{2}{*}{ 悹 } & \multirow{2}{*}{ 产 } & \multirow{2}{*}{ 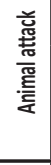 } & \multirow{2}{*}{ 要 } \\
\hline & $\stackrel{\Sigma}{\Sigma}$ & 䛵 & $\frac{\text { 음 }}{\mathrm{J}}$ & $\begin{array}{l}\text { 竧 } \\
\text { 离 } \\
\text { 离 }\end{array}$ & 胥 & "ᄒ⿱宀 & 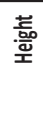 & & & & & & & \\
\hline 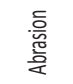 & $\begin{array}{l}\text { बे } \\
\text { o } \\
\stackrel{0}{\Rightarrow}\end{array}$ & $\begin{array}{l}\text { 응 } \\
\text { o } \\
\text { o }\end{array}$ & 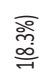 & 0 & 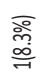 & 0 & 0 & 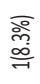 & 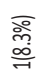 & 0 & 0 & 0 & 0 & 产 \\
\hline 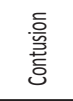 & $\begin{array}{l}\text { बे } \\
\text { ले }\end{array}$ & 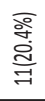 & $\begin{array}{l}\overline{\text { a }} \\
\stackrel{m}{\sim}\end{array}$ & $\begin{array}{l}\overline{\text { gे }} \\
\stackrel{y}{y}\end{array}$ & $\begin{array}{l}\text { बa } \\
\frac{\mathrm{g}}{m} \\
m\end{array}$ & $\begin{array}{l}\text { 을 } \\
\text { Е }\end{array}$ & 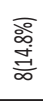 & 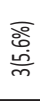 & 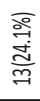 & 0 & 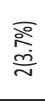 & $\begin{array}{l}\overline{\bar{a}} \\
\text { m } \\
\bar{\sim}\end{array}$ & 0 & 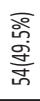 \\
\hline 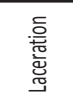 & $\begin{array}{l}\overline{8} \\
\text { 离 } \\
\text { in }\end{array}$ & $\begin{array}{l}\overline{\mathrm{g}} \\
\text { J } \\
\text { gे }\end{array}$ & $\begin{array}{l}\bar{\alpha} \\
\text { مे } \\
\text { oे }\end{array}$ & $\begin{array}{l}\overline{\mathrm{g}} \\
\stackrel{\mathrm{J}}{=}\end{array}$ & 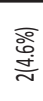 & $\begin{array}{l}\bar{\sigma} \\
\text { ले } \\
\text { gे }\end{array}$ & 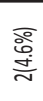 & 0 & 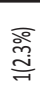 & 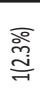 & 0 & $\begin{array}{l}\bar{\alpha} \\
\text { న্ }\end{array}$ & $\begin{array}{l}\overline{\mathrm{d}} \\
\stackrel{ }{=}\end{array}$ & 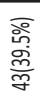 \\
\hline 栗 & 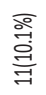 & $\frac{\overline{\frac{a}{9}}}{\frac{m}{m}}$ & $\begin{array}{l}\bar{d} \\
\dot{g} \\
\frac{g}{N}\end{array}$ & 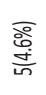 & $\begin{array}{l}\text { 总 } \\
\text { 赑 }\end{array}$ & $\begin{array}{l}\text { 豆 } \\
\text { if }\end{array}$ & 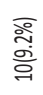 & 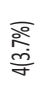 & 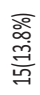 & $\begin{array}{l}\text { 용 } \\
\text { 음 }\end{array}$ & 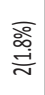 & $\begin{array}{l}\text { बig } \\
\text { o } \\
\text { in }\end{array}$ & $\begin{array}{l}\text { बे } \\
\text { อे }\end{array}$ & 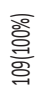 \\
\hline
\end{tabular}

Table V: Pattern of associated soft tissue injury according to mechanism of injury

\begin{tabular}{|c|c|c|c|c|c|c|c|c|c|c|c|c|c|c|}
\hline \multirow{2}{*}{ 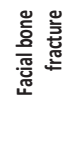 } & \multicolumn{4}{|c|}{ RTA } & \multicolumn{3}{|c|}{ Fall } & \multirow{2}{*}{ 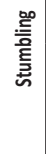 } & \multirow{2}{*}{ 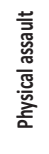 } & \multirow{2}{*}{ 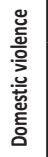 } & \multirow{2}{*}{ 产 } & \multirow{2}{*}{ 产 } & \multirow{2}{*}{ 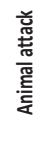 } & \multirow{2}{*}{ 胥 } \\
\hline & $\sum_{\Sigma}^{\top}$ & 嘀 & 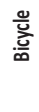 & 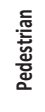 & 足 & "ᄒ⿱ & 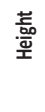 & & & & & & & \\
\hline \multicolumn{15}{|l|}{ Karnali } \\
\hline Dolpa & 0 & 0 & 0 & 0 & 0 & 0 & 0 & 0 & 0 & 0 & 0 & 0 & 0 & 0 \\
\hline Humla & 0 & 0 & 0 & 0 & 0 & 0 & 0 & 0 & 0 & 0 & 0 & 0 & 0 & 0 \\
\hline Jumla & 0 & 0 & 0 & 0 & 0 & 0 & 1 & 0 & 0 & 0 & 0 & 0 & 0 & 1 \\
\hline Kalikot & 0 & 1 & 0 & 0 & 0 & 0 & 0 & 1 & 1 & 0 & & 0 & 0 & 3 \\
\hline Mugu & 0 & 0 & 0 & 0 & 0 & 0 & 0 & 0 & 0 & 0 & 0 & 0 & 0 & 0 \\
\hline \multicolumn{15}{|l|}{ Bheri } \\
\hline Banke & 1 & 5 & 1 & 0 & 0 & 1 & 0 & 0 & 2 & 1 & 0 & 0 & 0 & 11 \\
\hline Bardiya & 0 & 1 & 2 & 0 & 0 & 0 & 0 & 0 & 2 & 0 & 1 & 1 & 0 & 7 \\
\hline Surkhet & 1 & 0 & 0 & 2 & 1 & 0 & 0 & 1 & 0 & 0 & 0 & 0 & 0 & 5 \\
\hline Dailekh & 0 & 0 & 0 & 0 & 2 & 1 & 1 & 0 & 1 & 0 & 0 & 0 & 0 & 5 \\
\hline Jajarkot & 0 & 0 & 0 & 0 & 0 & 0 & 2 & 0 & 0 & 0 & 0 & 0 & 0 & 2 \\
\hline \multicolumn{15}{|l|}{ Rapti } \\
\hline Dang & 1 & 5 & 0 & 1 & 0 & 0 & 1 & 0 & 1 & 0 & 0 & 0 & 0 & 9 \\
\hline Salyan & 1 & 0 & 0 & 0 & 0 & 0 & 1 & 0 & 0 & 0 & 0 & 0 & 0 & 2 \\
\hline Rolpa & 1 & 0 & 0 & 0 & 0 & 0 & 0 & 0 & 0 & 0 & 0 & 1 & 0 & 2 \\
\hline Pyuthan & 0 & 2 & 0 & 0 & 0 & 0 & 0 & 0 & 0 & 0 & 0 & 0 & 0 & 2 \\
\hline Rukum & 0 & 1 & 0 & 1 & 0 & 0 & 0 & 0 & 1 & 0 & 0 & 0 & 0 & 3 \\
\hline \multicolumn{15}{|l|}{ Seti } \\
\hline Bajhang & 0 & 0 & 0 & 0 & 1 & 0 & 1 & 0 & 1 & 0 & 0 & 0 & 0 & 3 \\
\hline Bajura & 0 & 0 & 0 & 0 & 0 & 0 & 0 & 0 & 0 & 0 & 0 & 0 & 1 & 1 \\
\hline Doti & 0 & 0 & 0 & 0 & 0 & 0 & 0 & 0 & 0 & 0 & 0 & 0 & 0 & 0 \\
\hline Achham & 0 & 1 & 0 & 0 & 0 & 1 & 1 & 0 & 1 & 0 & 0 & 0 & 0 & 4 \\
\hline Kailai & 2 & 10 & 2 & 1 & 0 & 1 & 1 & 0 & 2 & 0 & 0 & 1 & 0 & 20 \\
\hline \multicolumn{15}{|l|}{ Mahakali } \\
\hline Darchula & 0 & 0 & 0 & 0 & 1 & 0 & 0 & 0 & 0 & 0 & 0 & 1 & 0 & 2 \\
\hline Baitadi & 0 & 0 & 0 & 0 & 0 & 0 & 0 & 1 & 0 & 0 & 0 & 0 & 0 & 1 \\
\hline \multicolumn{15}{|l|}{ Dadeldhura } \\
\hline Kanchanour & 0 & 2 & 0 & 0 & 0 & 0 & 0 & 0 & 1 & 0 & 1 & 0 & 0 & 4 \\
\hline
\end{tabular}

Table VI: Geographical distribution of Maxillofacial fractures according to the etiology of injuries 
The mean duration of hospital stay is 6.29 days (1-27 days) and median and mode 4 days.

RTA is more common in terai region such as: Kailali and Dang. Fall injuries are more common in mountain and hilly districts such as: Achham, Dailekh, Jumla, Jajarkot, Bajhang, Salyan, Darchula. (Table VI)

\section{DISCUSSION}

The most common reported etiology of maxillofacial fracture in our study is road traffic accident. Motorbike accident accounted for majority of fracture in our study similar to study conducted in western part of Nepal by Rajib Khadka et $\mathrm{al}^{11}$ which is in contrast with the studies by B. van den Bergh et $\mathrm{a}^{12}$ bicycle; Miguel S. Cabalag et $\mathrm{a}^{5}$ and P. Boffano et $\mathrm{al}^{4}$ assault, which may be due to the sociocultural and legislative differences between these countries. The maxillofacial injuries occurred most commonly in male with a ratio of 5.2:1 and in third decade of life which is in agreement with several other studies $3,6,7,12$. We reported a higher male predilection than studies conducted in other part of Nepal ${ }^{8,10,12}$. In European Maxillofacial Trauma (EURMAT) population maxillofacial Injury was more in $4^{\text {th }}$ decade of life ${ }^{13}$ which can be due to increasingly active elder population. We also reported a case of domestic violence which could be the representative case in our society.

We reported 33 cases with associated injuries with maxillofacial trauma where head injury (23\%) was most common followed by injury to the extremities $(10.3 \%)$. The frequency of associated injuries varies from 14-56\% among different studies with many author reporting head injury being the most commonly reported injury ${ }^{7}$. Motorbike accident (12 cases) was the most common cause followed by pedestrian for associated injuries in our study. This finding is in contrast to study by Mohammad Zandi $^{3}$ and Krishna $\mathrm{KC}^{8}$ who reported extremities injury and MVA to be the most common. C-spine fracture was reported in five cases (5.7\%) with bicycle, pedestrian, fall and occupational injuries as etiology showing the necessity to rule out C- spine injury in all cases of maxillofacial injury. The average incidence of soft tissue injury was 1.25 /patient. Contusion was the most common type of soft tissue injury followed by laceration and abrasion. This finding is in contrast with study by Mohammad Zandi ${ }^{3}$ who reported laceration to be the most common type of soft tissue injury followed by abrasion, contusion and avulsion. This could be due to the fact that we only reported the soft tissue injury in patient reporting with maxillofacial fractures.

We reported a total of 157 fractures in 87 patient accounting for 1.8 fractures/patient. Mandible fracture is the most common fracture followed by ZMC, Maxilla, Palatal split, nasal, Le-Fort III, Le-Fort I and II. Based on the anatomic location we reported Parasymphysis (40.2\%), Angle (22.7\%), Symphysis (13.4\%), body $(11.3 \%)$, condyle $(10.3 \%)$ and ramus $(2.1 \%)$ fracture. Left parasymphis was more commonly fractured than right side whereas, body, angle, ramus, and condyle were fractured more commonly on right side. Our finding agrees with the finding of study by Ram Bhakta Adhikari that parasymphysis is more commonly involved site in mandibular fracture but we reported more frequency of angle and symphysis fracture in contrast to his study ${ }^{10}$.

ZMC fracture is the second most common site of maxillofacial fracture similar to study by Krishna $\mathrm{KC}^{8}$. But our study differ from that of the study by Mohammad Zandi ${ }^{3}$ where nasal bone was the most commonly fractured bone and Le-Fort I fracture was more common than Le-Fort II and III and Miguel S. Cabalog ${ }^{5}$ reported orbital fracture to be the most common fracture. This variation could be because of the differences in sample of the study and method of collection of data. In our setup isolated nasal bone fracture are mostly managed by ENT department excluding it from the study. We also reported prevalence of fall from tree and stumbling as a separate etiology for maxillofacial injury similar to study by Ramané Béogo ${ }^{6}$, and Mohammad Zandi ${ }^{3}$. The etiology fall from tree and height is related to search for wood and grass. We also reported fall from height mainly in first to third decade of life which is mainly because of slippery road in villages and decrease safety regulation on school for students who have access to the roof. Physical assault is seen more common in second to third decade of life as reported in other studies $6,12,13$.

We have reported the maxillofacial injuries based on geographical location with hilly district reporting higher incidence of injuries due to fall. This is because of the topographical location of this district and dependency of the people of that region in forest for fuel and feeding cattles. We have not found any study till date showing incidence of maxillofacial injuries based on geographical location in our country.

\section{CONCLUSION}

Male patient in their third decade mostly had mandible fracture due to road traffic accident. Head injury was the most common associated injury. Contusion was the most common type of associated soft tissue injury. The result of this study suggests that it is high time to implement injury prevention strategy in our country beginning from strong implementation of traffic rules, increasing the quality of roads, injury prevention strategy in school, sports safety regulations, occupational safety regulations, safety of villages near national parks, to education of youth and general public in social norms and values.

\section{REFERENCES}

1. Martinez AY, Como JJ, Vacca M, Nowak MJ, Thomas CL, Claridge JA. Trends in Maxillofacial Trauma: A Comparison of Two Cohorts of Patients at a Single Institution 20 Years Apart. J Oral Maxillofac Surg. 2010;72(4):750-4.

2. Subedi S, Shrestha B, Rb A. Pattern of Maxillofacial fracture in Western and Central Nepal: An experience in 3 tertiary level health institutions. 2014;(3):8-13.

3. Zandi M, Khayati A, Lamei A, Zarei H. Maxillofacial injuries in western Iran: A prospective study. Oral Maxillofac Surg. 2011;15(4):201-9. 
4. Boffano P, Roccia F, Zavattero E, Dediol E, Ugle V, Stephens J, et al. European Maxillofacial Trauma ( EURMAT) project : A multicentre and prospective study. 2015;43:62-70.

5. Cabalag MS, Wasiak J, Andrew NE, Tang J, Kirby JC, Morgan DJ. Epidemiology and management of maxillofacial fractures in an Australian trauma centre. J Plast Reconstr Aesthetic Surg [Internet]. 2014;67(2):183-9. Available from: http://dx.doi. org/10.1016/j.bjps.2013.10.022

6. Béogo R, Dakouré PW, Coulibaly TA, Donkor P. Epidemiology of facial fractures: an analysis of 349 patients. Médecine Buccale Chir Buccale. 2014;20(1):13-6.

7. Rezaei M, Jamshidi S, Jalilian T, Falahi N. Epidemiology of maxillofacial trauma in a university hospital of Kermanshah, Iran. J Oral Maxillofac Surgery, Med Pathol [Internet]. 2017;29(2):110-5. Available from: http://dx.doi.org/10.1016/j. ajoms.2016.09.008

8. K.C. K, Shrestha JM. Maxillofacial injuries managed at Tribhuvan University Teaching Hospital, Kathmandu, Nepal: a 7 year retrospective study. J Soc Surg Nepal. 2016;19(1):4-8.

9. Yadav SK, Mandal BK, Karn A, Sah AK. Maxillofacial trauma with head injuries at a tertiary care hospital in Chitwan, Nepal: Clinical, medico-legal, and critical care concerns. Turkish J Med Sci. 2012;42(SUPPL.2):1505-12.

10. Adhikari RB, Karmacharya A MN. Pattern of mandibular fractures in western region of Nepal. Nepal J Med Sci. 2012;1(1):45-8.

11. Chaurasia N, Khadka R. Four years prospective study of the maxillofacial trauma at a tertiary center in Western Nepal. J Orofac Sci. 2014;6(2):78.

12. Van Den Bergh B, Karagozoglu KH, Heymans MW, Forouzanfar T. Aetiology and incidence of maxillofacial trauma in Amsterdam: A retrospective analysis of 579 patients. J Cranio-Maxillofacial Surg [Internet]. 2012;40(6):e165-9. Available from: http:// dx.doi.org/10.1016/j.jcms.2011.08.006

13. Boffano $P$, Roccia $F$, Zavattero $E$, Dediol $E$, Uglešić $V$, Kovačič Ž, et al. European Maxillofacial Trauma (EURMAT) project: A multicentre and prospective study. J Cranio-Maxillofacial Surg. 2015;43(1):62-70. 